\title{
Circulating free DNA, new dynamic marker in nonsmall cell lung cancer patients?
}

\author{
Jordi Remon ${ }^{1}$, Julie Gorham², Benjamin Besse ${ }^{1,3}$ and Jean-Paul Sculier ${ }^{2}$ \\ Affiliations: ${ }^{1}$ Medical Oncology Dept, Gustave Roussy, Villejuif, France. ${ }^{2}$ Oncologic Intensive Care and \\ Emergencies and Thoracic Oncology Dept, Institut Jules Bordet (ULB), Brussels, Belgium. ${ }^{3}$ Medical Dept, \\ Paris Sud University, Paris, France.
}

Correspondence: Jordi Remon, Medical Oncology Department, Villejuif, France.

E-mail: jordi.remon-masiplagustaveroussy.fr

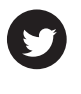

@ERSpublications

cfDNA concentration might be a prognostic factor in NSCLC and could be a tool for detecting molecular alterations http://ow.ly/TF8TC

Platinum-based doublet chemotherapy prolongs survival and improves quality of life as first-line treatment for non-selected and good performance status patients with advanced nonsmall cell lung cancer (NSCLC) [1]. However, the survival benefit is limited, even if this combination is the standard of care for decades, no clinically relevant predictive biomarkers have been discovered so far. The identification of surrogates for survival is an important topic for cancer therapy selection. Indeed, overall survival is still the best criterion for predicting treatment efficacy in lung cancer but it can, of course, not be used in clinical practice. In a recent systematic review of the literature, some intermediate criteria have been shown to be potentially useful predictors such as time to progression, progression-free survival, objective response, local control after radiotherapy, downstaging in locally advanced NSCLC, complete resection and pathological TNM (tumour, node, metastases) in resected NSCLC or circulating molecular markers or cells. Retrospective studies have suggested that carcinoembryonic antigen, cytokeratin 19 fragments, pro-gastrin-releasing peptide and, to a lesser extent, neuron specific enolase, cancer antigen 125 and cancer antigen 19-9, used as single criterion to assess overall survival, could be adequate intermediate criteria for survival in lung cancer patients [2]. The use of more sophisticated molecular markers developed thanks to recent progresses in molecular biology has also so far not been successful for that purpose even if high throughput techniques are used such as transcriptomic analyses assessing multiple miRNAs and mRNAs [3].

In the study reported in the present issue of the European Respiratory Journal, Tissot et al. [4] have assessed circulating cell-free DNA (cfDNA), which comprises small fragments of nucleic acids that are released from normal cells and tumours by programmed cell death (apoptosis). cfDNA can be detected and monitored in plasma of blood in all patients [5]. Thus, cfDNA has emerged as an attractive tumour marker for its minimal invasive, convenient, and easily accepted properties.

The purpose of the study was to determine if this biomarker can be used as a prognostic tool for tumour response assessment and for survival in NSCLC patients treated by platinum-based chemotherapy. The authors report that cfDNA concentration in plasma samples collected before (baseline) platinum-based chemotherapy in 218 locally advanced or metastatic NSCLC patients had an independent prognostic value. Patients with highest cfDNA concentration showed a significantly shorter progression-free survival $(p=0.034)$ and overall survival $(p=0.001)$ than patients with lower cfDNA concentrations. However, changes in total cfDNA concentration during treatment were not predictive to assess the effectiveness of chemotherapy in NSCLC patients. In terms of methodology, the main advantages of the study by TissoT et al. [4] are its prospective character, the multivariable analysis and the relatively inexpensive and uncomplicated method of dosage, easily implementable in clinical practice. But a reproducible cut-off determination is lacking, being a necessary requirement for external validation studies. Other weak points

Received: Sept 062015 | Accepted: Sept 072015

Conflict of interest: None declared.

Copyright OERS 2015 
of the study are the lack of a priori statistical considerations and the use of various chemotherapy regimens, even if all are platinum-based.

High cfDNA level has been proven to be a non-invasive biomarker of poor outcome in lung cancer patients [6-8], whereas other studies did not report such a relationship [9]. This is mostly explained by the lack of standardisation of the cfDNA test, with major differences regarding cfDNA extraction, quantification and cut-off points. Moreover, another limitation is linked to the source of cfDNA: its concentration reflects not only changes in circulating tumour DNA (ctDNA) but also reflect medical conditions or patients' characteristics that may lead to an increase in cfDNA concentration [10]. Also molecular characteristics of the tumours might have an impact in the prognostic value of cfDNA concentration [11]. Tissot et al. [4] reported a significant positive association between baseline cfDNA concentration and Eastern Cooperative Oncology Group performance status at diagnostic (higher cfDNA concentration in worse performance status; $\mathrm{p}=0.001$ ), but not with stage or histological type. This suggests that patients' characteristics might influence in the basal cfDNA concentration, which could have an impact in the final prognosis.

In fact, cfDNA translates an inflammatory process. Simples scores based on routine tests have been developed to determine the prognostic and predictive role of inflammation in oncology, with many studies conducted for lung cancer [12]. The modified Glasgow score is the mostly used for clinically assessing inflammation [13]. This score includes: C-reactive protein (CRP), and albumin to define three groups of different prognosis: 0 in the absence of an elevated CRP, 1 being an increased CRP and 2 an increased CRP and a decreased albumin [14]. It has been assessed as a useful and important predictor of cancer-specific survival in NSCLC patients [15]. This score might be used as a stratifying factor when assessing new biomarkers related to inflammation such as cfDNA. Interestingly, and contrary to data obtained by Tіssот et al. [4] with cfDNA, the modified Glasgow score also predicted response and toxicity to chemotherapy in a prospective study in patients with metastatic NSCLC [16].

Because cell-free ctDNA is a potential surrogate for the entire tumour genome, cfDNA has been extensively studied as an alternative to biopsy, leading the term "liquid biopsy" [17]. The treatment of lung cancer is increasingly guided by molecular subtyping, such as mutations in the epidermal growth factor receptor (EGFR) gene [18], but access to tumour tissue to perform the molecular test sometimes is limited. The detection of ctDNA allows the easy identification of druggable genomic alterations, with high concordance, specificity, and sensitivity such as demonstrated with the detection of EGFR mutation [19-21]. Despite the initial dramatic response rate and benefit in progression free survival to EGFR tyrosine kinase inhibitors (TKI), most of EGFR mutant NSCLC patients develop acquired resistance 1 year after treatment initiation [22], the T790M mutation being the main mechanism of resistance. Personalised second-line therapy in EGFR mutant NSCLC patients with AZD9291 [23] or CO1686 [24] requires testing the T790M status in a recent tissue biopsy, being a predictive factor for these new therapeutic strategies. Unfortunately, the location of the tumour and the risk of complications are serious limitations to re-biopsies in NSCLC [25] and not all patients are willing to perform it. Qualitative and quantitative T790M in plasma ctDNA provide a non-invasive and sensitive assay to predict prognosis of EGFR-TKI treatment [26] and treatment strategy. There are clear advantages to measuring ctDNA as a marker of tumour dynamics over conventional protein biomarkers or even imaging studies. For one, ctDNA has a comparatively short half-life (approximately $2 \mathrm{~h}$ ), allowing for evaluation of tumour changes in hours rather than weeks to months [27]. Also, ctDNA may be more representative of tumour heterogeneity than a small biopsy sample and could enable the investigation of acquired resistance mechanisms and allows the investigators to monitor the evolution of resistance over the time [28].

We could conclude by Tissot et al. [4] study that total cfDNA concentration appears to be an independent prognostic factor in lung cancer but the most appealing application regarding cfDNA, so far, is the detection and dynamic monitoring of molecular alterations. Nevertheless, the establishment of robust and standardised protocols for blood sampling, processing, storage, DNA extraction and analysis, and cut-off points to define the clinical relevance of the findings are required before cfDNA biomarkers can be utilised in clinical practice.

\section{References}

1 NSCLC Meta-Analyses Collaborative Group. Chemotherapy in addition to supportive care improves survival in advanced non-small-cell lung cancer: a systematic review and meta-analysis of individual patient data from 16 randomized controlled trials. J Clin Oncol 2008; 26: 4617-4625.

2 Berghmans T, Pasleau F, Paesmans M, et al. Surrogate markers predicting overall survival for lung cancer: ELCWP recommendations. Eur Respir J 2012; 39: 9-28.

3 Berghmans T, Ameye L, Lafitte J-J, et al. Prospective Validation Obtained in a Similar Group of Patients and with Similar High Throughput Biological Tests Failed to Confirm Signatures for Prediction of Response to Chemotherapy and Survival in Advanced NSCLC: A Prospective Study from the European Lung Cancer Working Party. Front Oncol 2014; 4: 386. 
Tissot C, Toffart A-C, Villar S, et al. Circulating free DNA concentration is an independent prognostic biomarker in lung cancer. Eur Respir J 2015; 46: 1773-1780.

5 Francis G, Stein S. Circulating Cell-Free Tumour DNA in the Management of Cancer. Int J Mol Sci 2015; 16: $14122-14142$.

6 Sirera R, Bremnes RM, Cabrera A, et al. Circulating DNA is a useful prognostic factor in patients with advanced non-small cell lung cancer. J Thorac Oncol 2011; 6: 286-290.

7 Gautschi O, Bigosch C, Huegli B, et al. Circulating deoxyribonucleic Acid as prognostic marker in non-small-cell lung cancer patients undergoing chemotherapy. J Clin Oncol 2004; 22: 4157-4164.

8 Sozzi G, Roz L, Conte D, et al. Plasma DNA quantification in lung cancer computed tomography screening: five-year results of a prospective study. Am J Respir Crit Care Med 2009; 179: 69-74.

9 Kumar S, Guleria R, Singh V, et al. Efficacy of circulating plasma DNA as a diagnostic tool for advanced non-small cell lung cancer and its predictive utility for survival and response to chemotherapy. Lung Cancer 2010; 70: 211-217.

10 Ilie M, Hofman V, Long E, et al. Current challenges for detection of circulating tumor cells and cell-free circulating nucleic acids, and their characterization in non-small cell lung carcinoma patients. What is the best blood substrate for personalized medicine? Ann Transl Med 2014; 2: 107.

11 Karachaliou N, Mayo-de Las Casas C, Queralt C, et al. Association of EGFR L858R Mutation in Circulating Free DNA With Survival in the EURTAC Trial. JAMA Oncol 2015; 1: 149-157.

12 Proctor MJ, Morrison DS, Talwar D, et al. A comparison of inflammation-based prognostic scores in patients with cancer. A Glasgow Inflammation Outcome Study. Eur J Cancer 2011; 47: 2633-2641.

13 McMillan DC. The systemic inflammation-based Glasgow Prognostic Score: a decade of experience in patients with cancer. Cancer Treat Rev 2013; 39: 534-540.

14 McMillan DC, Crozier JEM, Canna K, et al. Evaluation of an inflammation-based prognostic score (GPS) in patients undergoing resection for colon and rectal cancer. Int J Colorectal Dis 2007; 22: 881-886.

15 Leung EYL, Scott HR, McMillan DC. Clinical utility of the pretreatment glasgow prognostic score in patients with advanced inoperable non-small cell lung cancer. J Thorac Oncol 2012; 7: 655-662.

16 Gioulbasanis I, Pallis A, Vlachostergios PJ, et al. The Glasgow Prognostic Score (GPS) predicts toxicity and efficacy in platinum-based treated patients with metastatic lung cancer. Lung Cancer 2012; 77: 383-388.

17 Heitzer E, Ulz P, Geigl JB. Circulating tumor DNA as a liquid biopsy for cancer. Clin Chem 2015; 61: 112-123.

18 Kris MG, Johnson BE, Berry LD, et al. Using multiplexed assays of oncogenic drivers in lung cancers to select targeted drugs. JAMA 2014; 311: 1998-2006.

19 Luo J, Shen L, Zheng D. Diagnostic value of circulating free DNA for the detection of EGFR mutation status in NSCLC: a systematic review and meta-analysis. Sci Rep 2014; 4: 6269.

20 Douillard J-Y, Ostoros G, Cobo M, et al. Gefitinib treatment in EGFR mutated caucasian NSCLC: circulating-free tumor DNA as a surrogate for determination of EGFR status. J Thorac Oncol 2014; 9: 1345-1353.

21 Mok T, Wu Y-L, Lee JS, et al. Detection and Dynamic Changes of EGFR Mutations from Circulating Tumor DNA as a Predictor of Survival Outcomes in NSCLC Patients Treated with First-line Intercalated Erlotinib and Chemotherapy. Clin Cancer Res 2015; 21: 3196-3203.

22 Normando SRC, Cruz FM, Del Giglio A. Cumulative meta-analysis of epidermal growth factor receptor-tyrosine kinase inhibitors as first-line therapy in metastatic non-small-cell lung cancer. Anticancer Drugs 2015.

23 Jänne PA, Yang JC-H, Kim D-W, et al. AZD9291 in EGFR inhibitor-resistant non-small-cell lung cancer. $N$ Engl J Med 2015; 372: 1689-1699.

24 Sequist LV, Soria J-C, Goldman JW, et al. Rociletinib in EGFR-mutated non-small-cell lung cancer. $N$ Engl J Med 2015; 372: 1700-1709.

25 Bosc C, Ferretti GR, Cadranel J, et al. Rebiopsy during disease progression in patients treated by TKI for oncogene-addicted NSCLC. Target Oncol 2015; 10: 247-253.

26 Wang Z, Chen R, Wang S, et al. Quantification and dynamic monitoring of EGFR T790M in plasma cell-free DNA by digital PCR for prognosis of EGFR-TKI treatment in advanced NSCLC. PloS One 2014; 9: e110780.

27 Diaz LA, Bardelli A. Liquid biopsies: genotyping circulating tumor DNA. J Clin Oncol 2014; 32: 579-586.

28 Oxnard GR, Paweletz CP, Kuang Y, et al. Noninvasive detection of response and resistance in EGFR-mutant lung cancer using quantitative next-generation genotyping of cell-free plasma DNA. Clin Cancer Res 2014; 20: 1698-1705. 